\title{
Advances in fiber optic DNA-based sensors: A Review
}

\author{
Desiree Santano, Aitor Urrutia, Carlos R. Zamarreño, Senior Member, IEEE, Ignacio Del Villar
}

\begin{abstract}
DNA is becoming increasingly important in the domain of optical fiber sensors, either as a tool for biosensing, or as a target to detect. In this review the main contributions of the last years are presented both in the domain of wavelength and intensity based configurations. This review comprises the use of natural single strand DNA (ssDNA) sequences as receptors for the detection of SsDNA sequences through hybridization, synthetic nucleic acids receptors for detection of complementary ssDNA sequences, and sensors based on natural and synthetic SSDNA receptors used for the detection of non-DNA targets. Parameters such as sensitivity, detection range and limit of detection are analyzed and discussed in detail to the purpose of comparing the different technologies and knowing the future lines to follow in the domain of fiber optic DNA-based sensors.
\end{abstract}

Index Terms-Biosensors, DNA based sensors, nanostructured materials, optical fiber sensors.

\section{INTRODUCTION}

$\mathrm{F}$ IBER-OPTIC sensing technology has become increasingly mature and popular thanks to acceptable costs, compact instrumentation development, high accuracy, and the capability of performing measurements at inaccessible sites, over large distances, in strong magnetic fields, and in harsh environments [1]. The dramatic growth that optical fiber technology has experienced in the recent decades can also be explained by the progressive introduction of this technology in the field of medicine. Nowadays, it is quite common to find optical fibers in interesting applications such as endoscopy, laser applications allowing the removal of varicose veins, or to protect elderly men from benign prostatic hyperplasia (BPH) [2].

In the field of optical fiber sensors, there are two main detection methods: intensity and wavelength (polarization measurements are also used in very specific applications) detection based sensors [3], [4]. The first one is based on monitoring light intensity changes at a specific wavelength range. Here, absorption and luminescence are the most widely used techniques [5]. They are quite simple to manage and, therefore, the most cost-effective. However, they have a major drawback, which is the need for a stable signal reference,

This work was supported in part by the Spanish Agencia Estatal de Investigación (AEI) (PID2019-106231RB-I00), the Public University of Navarra (PJUPNA26), and $\mathrm{PhD}$ research grants. In addition, this project has received funding from the ATTRACT call financed by the European Union's Horizon 2020 research and innovation program under grant agreement No 777222 .

D. Santano, A. Urrutia, C. Ruiz-Zamarreño, and I. Del Villar are with the IEEC Department, Public University of Navarra, Pamplona, 31006 SPAIN (corresponding author: ignacio.delvillar@ unavarra.es).

A. Urrutia, C. Ruiz-Zamarreño, and I. Del Villar are with the Institute of Smart Cities (ISC), Public University of Navarra, Pamplona, 31006 SPAIN. since the sources may vary their intensity as a function of time. Some techniques have been developed for compensating this issue. The simplest technique consists of providing a reference signal to compare with and thus eliminate the intensity variations in the source [6].

The second method is based on wavelength detection. In particular, it is based on tracking the wavelength shift of the transmission or attenuation bands in the spectrum. These configurations are usually more robust with respect to intensity-based techniques, since they avoid the instability of optical intensity signals. However, they are more expensive, since they require the utilization of a spectrometer or an interrogator to monitor the wavelength shift [2].

Concerning the structure of optical fiber sensing platforms, they basically consist of three clearly defined parts: the substrate, the interface and the receptors (see Abstract Figure). The substrate transduces the interactions between the receptor and the antigen into measurable variables and converts them into an optical signal that can be processed afterwards. Regarding the interface, it contains one or several layers devoted to functionalize the surface of the substrate to enable the attachment of the receptors (optionally, between the interface and the substrate, an additional layer or a stack of layers is included to excite one or several resonances).

In this review the interest is focused on the use of deoxyribose nucleic acids (DNA) as receptors. That includes natural single strand DNA (ssDNA) short sequences and DNA based synthetic structures. Natural nucleic acids are biomolecules that play a vital role in numerous processes in cells from the storage, transmission and expression of genetic information to regulatory roles [7]. Nucleic acids are complex organic substances whose molecules consist of many nucleotides linked in a long chain. Each nucleotide consists of the union of three components: a 5-carbon sugar (deoxyribose 
in DNA or ribose in ribonucleic acids - RNA), a phosphate group, and a nitrogenous base. The nitrogenous base of the nucleotides varies conforming the four different nucleotides of the DNA: adenine, guanine, cytosine, or thymine. The genetic information of the organisms is written in the sequence of nucleotides.

The DNA forms naturally a double helix linked by hydrogen bonds between the complementary bases, adenine and thymine or cytosine and guanine. In molecular biology, hybridization consisted in the spontaneous pairing of complementary ssDNA sequences by hydrogen bonds to create double stranded DNA (dsDNA) molecules. In addition, the short sequences of nucleotides are called oligonucleotides.

Natural nucleic acids, including DNA, can be applied as biological recognition elements in biodetection against various targets [8]. The specific base pairing between two strands of the nucleic acids can be used to detect complementary chains, giving rise to one of the most promising techniques to be investigated. Owing to their specificity, the detection of specific sequences of ssDNA can be used to detect genetic mutations or biomarkers associated with particular diseases [9]. The unique pattern of even short oligomers represents a powerful tool in terms of biosensing, because a single mismatch of a nucleobase can cause large changes in the affinity between two complementary strands [10].

Moreover, in recent decades, the advances in the genetic engineering allows the design of synthetic receptors which mimic natural molecule, such as peptide nucleic acids (PNA) DNA analogues or aptamers, molecular beacons (MBs) and DNA origami engineering designed DNA structures[11]-[13].

The PNAs are synthetic DNA analogues in which the phosphodiester backbone is replaced by repetitive units of $\mathrm{N}$ (2-ami-noethyl) glycine to which the purine and pyrimidine bases are attached via a methyl carbonyl linker. The synthetic backbone provides PNA with unique hybridization characteristics for the detection of the complementary ssDNA (cssDNA) sequence [14]. Even if their use is not widely extended in fiber optic sensing technology, PNA are well known in biosensing field.

Aptamers are short ssDNA or RNA molecules that own the capability to bind tightly and specifically to a wide range of targets such as proteins, peptides, amino acids, drugs, metal ions, and even whole cells. The sequence of the aptamer bases, known as the primary structure, is unique for each aptamer and will determine the affinity to the target molecule. The ability of the aptamers to form 2D and 3D complex structures is determined by the structure length and base pairing. The formation of unique secondary and tertiary structures confers the aptamers a high degree of specificity, which enables the detection of small molecules. The aptamer-target binding results from structure compatibility, stacking of aromatic rings, electrostatic and van der Walls interaction, hydrogen bonds or from a combination of these [9].

In the same way, MBs are another example of synthetic biorecognition elements based on nucleic acids that have been applied to fiber optic biosensing. A MB is a short oligonucleotide chain with a single-stranded loop and a hybridized stem structure. The stem consists of 5 to 7 nucleotides complementary to each other but, since it is short, it can readily dehybridize when a target oligonucleotide interacts with the longer loop strand. By attaching a fluorophore and a quencher to the ends of the beacon strand, it is possible to monitor the stem hybridization state by measuring the fluorescence emission from the fluorophore label. MBs have been successfully used in the detection of different sequences of DNA and RNA [15].

DNA origami is a process of molecular self-folding: a long single-stranded DNA (scaffold) is folded into prescribed objects by hundreds of short synthetic DNA oligonucleotides, which are designed to be complementary to different parts of the scaffold DNA [16]. This technique can be also implemented in optical fiber surfaces to develop promising devices [17], [18].In this review, we summarize the most relevant achievements in DNA and DNA analogues based optical fiber sensors of the last decade. The aim of this work is to provide an overview on the use of ssDNA receptors in optical fiber sensing (for the sake of clarity ssDNA sequences are not given in the text but in tables within each section that summarize all the works reported in this review). For this reason, we will consider optical fiber sensors that employ natural ssDNA sequences and synthetic receptors based on nucleic acids as receptors. The review is constructed as follows: Section 2 summarizes the main contributions based on the use of natural ssDNA sequences as receptors for the detection of ssDNA sequences through hybridization. In the section 3 contributions where synthetic nucleic acids receptors are employed in the detection of cssDNA sequences are considered. A later section 4 comprises sensors based on natural and synthetic ssDNA receptors used for the detection of non-DNA targets. Finally, discussion and some concluding remarks are presented in section 5 .

\section{SSDNA RECEPTORS FOR SSDNA TARGETS}

To date, a variety of methods including electrochemical and optical approaches have been applied to detect ssDNA specific sequences. This section presents the most recent advances in the utilization of optical fiber sensors for ssDNA detection using ssDNA as receptor. In general, short ssDNA sequences are used as receptor probes, to hybridize with a cssDNA target. Thus, those are sensors based on the ssDNA-cssDNA hybridization.

As stated in the introduction there are two major detection methods to measure changes within the optical fiber sensors: wavelength and intensity based sensors. In the next two subsections a summary of the main contributions in the domain of ssDNA-cssDNA hybridization within both groups is presented.

\section{A. Wavelength based sensors}

In the following lines, we will present the advances on wavelength based optical fiber sensors which employ natural ssDNA sequences for the detection of cssDNA. Various optical techniques and photonic structures have been proposed for ssDNA sensing purpose, such as SPR [19]-[21], localized surface plasmon resonance (LSPR) [22], [23], Fiber Bragg gratings [24]-[28] and interferometers [29]-[35].

The most well-known configuration is the surface plasmon resonance (SPR) in Kretschmann configuration, which is obtained with a metallic thin-film deposited on the substrate 
[36], a concept that was translated to optical fiber by Jorgenson et al [37]. SPR phenomenon occurs in the interface between a dielectric (optical fiber in this case) and a metallic thin-film (typically gold or silver) and it has been extensively used for sensing purposes. In particular, many efforts have been made to enhance the sensitivity of optical fiber SPR sensors in order to exploit them for the high-performance demanding biosensing applications [19]-[21].

A D-shape plastic optical fiber SPR biosensor based on a graphene and gold film has been proposed for detection of ssDNA-cssDNA hybridization process [21]. The sensor has experimentally demonstrated the detection of the hybridization and successfully distinguished single nucleotide variation. The refractive index (RI) sensitivity of this sensor is $1227 \mathrm{~nm} / \mathrm{RIU}$ in glucose RI solutions ranging from 1.333 to 1.3557 . The device also shows a linear response to the tested cssDNA concentration in the range $100 \mathrm{pM}-1 \mu \mathrm{M}$.

The detection of microorganisms is an interesting field from medical point of view. An optical fiber SPR biosensor was developed for the detection of Mycobacterium avium paratuberculosis and $M$. bovis bacterial sequences [20]. The bioassay was based on the hybridization/melting of ssDNAcoated gold nanoparticles on the optical fiber SPR sensor when targets are present. The sensor enables simultaneous quantification and identification of multiple cssDNA targets on the same platform. A detection range of 250-500 nM was successfully tested with this novel device.

In a different work, $400 \mu \mathrm{m}$ multimode fiber was sputtered with a $50 \mathrm{~nm}$ gold layer, which was then covered with a protein repulsive self-assembled monolayer of mixed polyethylene glycol [19]. The ssDNA coated SPR fibers were evaluated as a nucleic acid biosensor through a ssDNAcssDNA hybridization assay. A linear calibration curve was observed in the $0.5-5 \mu \mathrm{M}$ range with a limit of detection (LOD) of $2 \mathrm{nM}$.

In addition, LSPR sensors have been applied to test a wide range of biochemical interactions, such as ssDNA detection. The LSPR is a surface plasmon confined in a nanoparticle whose size is in the range of the light wavelength, which permits to enhance the sensitivity in the proximities of the nanoparticle, contrary to SPRs. However, according to the literature few works have recently reported optical fiber LSPR sensor based on hybridization [22], [23]. Jia et al. described a reflection LSPR fiber biosensor for ssDNA detection with gold nanospheres (AuNSs) and thiolated ssDNA as detecting probe on the tip of fiber [22]. As it is based on hybridization, the target is the cssDNA sequence. AuNSs of two different diameters (20 and $80 \mathrm{~nm}$ ) were used. The sensitivity of the sensors with $20 \mathrm{~nm}$ and $80 \mathrm{~nm}$ AuNSs was $82.86 \mathrm{~nm} / \mathrm{RIU}$ and $218.98 \mathrm{~nm} / \mathrm{RIU}$ respectively. The sensor with $80 \mathrm{~nm}$ AuNSs was used for the detection of 40 bases ssDNA-cssDNA hybridization detecting as low as $50 \mathrm{nM}$ target concentration.

Another study was proposed by Kaye et al, where an optical fiber LSPR hybridization based biosensor was created by constructing arrays of metallic nanostructures on the end facets of optical 4/125 $\mu \mathrm{m}$ core/cladding single mode fibers (SMF) utilizing nanofabrication technologies, including electron beam lithography and lift-off processes [23]. The obtained biosensor offers real-time, label-free, and lowsample-volume quantification of ssDNA concentrations in water. The LOD obtained by this optical sensor was $10 \mathrm{fM}$. Here, it is important to remark that this work achieves a significant improvement compared to previous works where a $50 \mathrm{nM}$ LOD was achieved. These results demonstrate the feasibility of LSPR based devices as compact biosensors for detection of ssDNA.

FBGs have also been used for the detection of ssDNAcssDNA hybridization. Bragg gratings are typically written in the core of an optical fiber to generate resonances in the optical transmission and reflection spectrum by coupling of light from the core mode to another co-propagating or counter-propagating mode that may be guided in the core or in the cladding of the optical fiber. On this basis, fiber Bragg gratings can be subdivided into two groups according to the grating period length: short-period fiber Bragg gratings, usually called fiber Bragg gratings (FBGs), and long-period fiber gratings (LPFGs) [38]. For FBGs, the periodicity of the
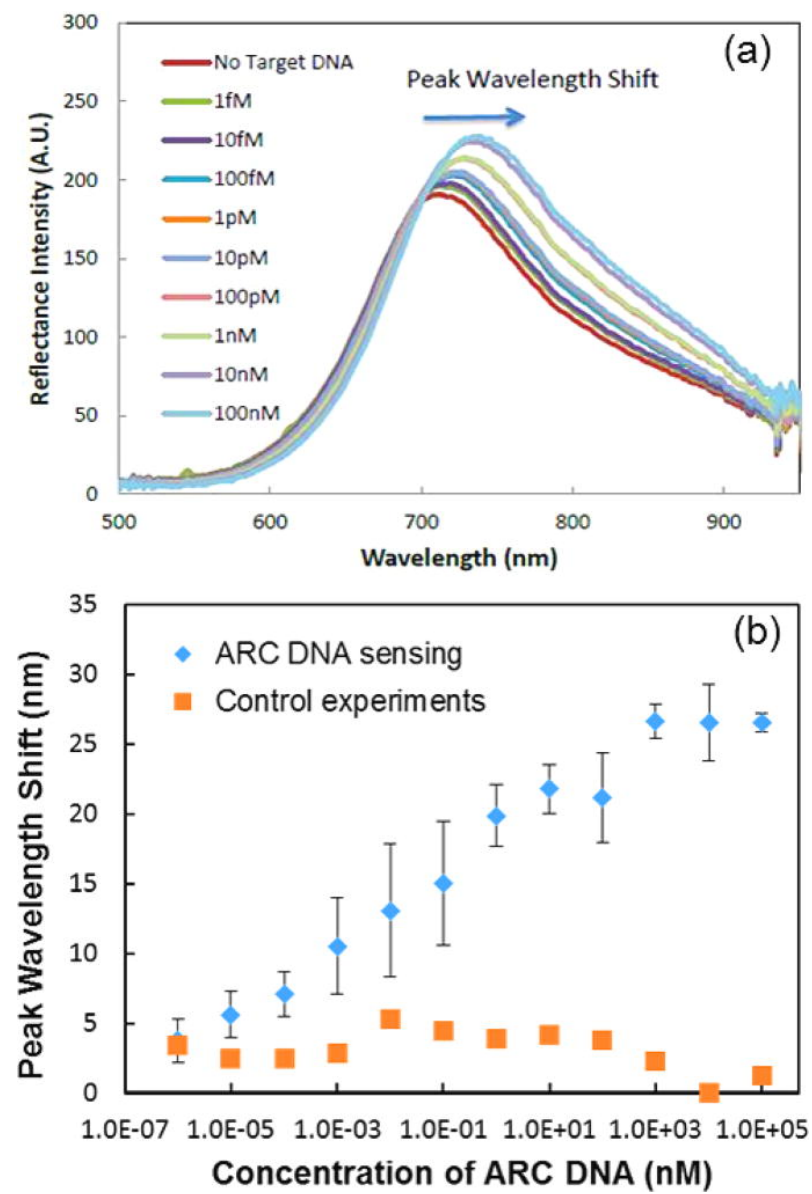

Fig. 1. (a) The representative reflectance spectra of the DNA detection assay with fiber optic LSPR. (b) LSPR peak wavelength shift due to the specific binding of target DNA on the Au nanodisk surfaces of the optical fiber end facet. The error bars represent the standard deviations calculated from three independent measurements. Reprinted with permission of Royal Society of Chemistry from [23]. (c) (2019)

grating structure is typically below $100 \mu \mathrm{m}$, whereas for LPFGs the periodicity ranges from $100 \mu \mathrm{m}$ to $1 \mathrm{~mm}$. In addition, Tilted Fiber Bragg gratings (TFBG) are those FBGs where the periodic variation of the core RI is induced by tilting the grooves of the fabrication mask by an angle $\theta$ with respect to the propagation axis [2]. 
Several approaches have been reported within LPG optical fiber technology. Regarding hybridization based biosensors, a study reported the successful detection of an ssDNA target at a concentration of $1 \mu \mathrm{M}$ [24]. The LPG pattern of the sensor was formed using a photolithograph process on the surface of a side-polished fiber. In addition, some years after it, a sensing platform that allows the discrimination between non-specific and specific binding even with just a single mismatch was published [25]. The biosensor consisted of a label-free fiber optic biosensor based on an LPG, and the experimental LOD obtained was $62 \mathrm{nM}$ ssDNA concentration.

One of the main issues of LPGs is the width of the resonances, typically in the range of tens of nanometers. However, the fabrication of a reduced bandwidth device of the order of $1 \mathrm{~nm}$ by using a high numerical aperture single mode fiber (SMF) allowed the improvement of the resolution of the LPGs. In particular, an LOD of $10 \mathrm{nM}$ ssDNA concentration was achieved with nanometric narrowband LPG sensors [26].

In addition, the generation of an SPR with a TFBG optical structure was reported for the detection of specific sequence of ssDNA [39]. The TFBG-SPR biosensor was exposed to two different concentrations of complementary ssDNA solutions, $0.1 \mathrm{mM}$ and $0.05 \mathrm{mM}$, which induced an increasing resonance wavelength shift, indicating that the hybridization with the complementary ssDNA molecules changed the effective refractive index in the vicinity of the golden layer.

Microfiber Bragg grating (mFBG) sensors have also been reported for the detection of ssDNA. MFBGs consist of a single straight Bragg grating inscribed in the silica microfiber, which provides two well-defined resonances in reflection mode. Both resonances show different response to external medium RI variations and present the same temperature sensitivity. Therefore, temperature- compensated RI measurement can be achieved by monitoring the wavelength separation between these two resonances. In this sense, two publications take advantage of the use of mFBG for ssDNAssDNA hybridization biosensing [27], [28]. In the first work, Sun et al. use a monolayer of poly-L-lysine functionalization for the label free biorecognition. The results show the real time detection of ssDNA for various concentrations of target ssDNA solutions, with a lowest detectable concentration of $0.5 \mathrm{mM}$. Moreover, specificity tests are performed with mismatched ssDNA sequences showing high specificity [28]. Later, the same group presented a reflective fiber optic biosensor based on mFBG for ssDNA-ssDNA hybridization detection using layer-by-layer self-assembly technology. Here, with the help of poly(ethylenimine), poly(acrylic acid) and ssDNA for the preparation of a polyelectrolyte multilayer film, the LOD was improved to $1 \mu \mathrm{M}$ ssDNA concentration [27].

Besides the mentioned types of sensors, there exist interferometric optical fiber sensors, which are based on the interference of light propagated through different optical paths, which is recombined and collected by an optical receptor. The resultant optical signal includes the contributions of two signals with different intensities and phase characteristics, which leads to the generation of an interference pattern. Depending on their optical configuration and structure, it is possible to classify them into four main types of fiber-based interferometers: Fabry-Perot, MachZehnder, Michelson, and Sagnac interferometers. Some of them can be analyzed by tracking intensity changes at a specific wavelength, whereas in others the interferometric fringe pattern is analyzed in the spectral and/or phase domain [38].

In recent studies, optical fiber interferometers have been used for the detection of some ssDNA sequences of microorganisms. In one of them, the application of photonic biosensor assays to diagnose the category-A select agent Francisella tularensis was investigated [29]. In addition, the specific detection and differentiation of $F$. tularensis subspecies tularensis (type A strain TI0902) and subspecies holarctica (type B strain LVS) was further accomplished using a single-mode multi-cavity fiber Fabry-Perot interferometric sensor. Detection was possible by the attachment of ssDNA probes directly to the fiber surface via layer-by-layer electrostatic self-assembly. Nanogram quantities of the cssDNA target of the two bacterial subespecies were detected, highlighting the sensitivity of this method for ssDNA detection without the use of the standard polymerase chain reaction (PCR) detection system.

Tapered optical fiber sensor has also been used as an interferometer for detecting a specific ssDNA sequence of Leptospira bacteria [33]. These bacteria cause leptospirosis, a deadly disease but with common early flu-like symptoms. The optical fiber taper was obtained stretching an optical SMF of diameter $125 \mu \mathrm{m}$. The taper was functionalized using (3aminopropyl) triethoxysilane prior to the receptor immobilization. The sensitivity of the obtained device was $1.2862 \mathrm{~nm} / \mathrm{nM}$ cssDNA concentration, which permitted to detect, with high specificity, cssDNA in concentrations as low as $0.1 \mathrm{fM}$.

Additionally, various papers describe the successful use of microfiber interferometers to detect ssDNA-cssDNA hybridization [30]-[32], [34]. In particular, a microfiber taper interferometer sensor was reported for cssDNA detection based on hybridization. Here, the taper was coated by a polymer showing a sensitivity of $7.24 \mathrm{~nm}$ per $\mu \mathrm{M}$ of cssDNA. In this case, the LOD was $100 \mathrm{pM}$ [30]. The same authors presented a microfiber based sensor using a graphene oxide coating deposited around the silica microfiber, which provides a strong interaction with the ssDNA target molecules. This modification leads to a larger wavelength shift of the optical interference fringe. Using this strategy, the LOD was improved down to 4.84 pM cssDNA solution [31]. The LOD obtained by other microfiber based sensors is within the same order of magnitude. For example, a microfiber Sagnac interferometric based sensor presented in [32] shows an LOD of 75 pM. A different sensor based on a microfiber in reflection configuration functionalized with a monolayer of poly-L-lysine also achieved a minimum detectable concentration of $10 \mathrm{pM}$ solution of cssDNA [34]. A summary of the previously reported works based on wavelength detection is presented in Table 1.

\section{B. Intensity based sensors}

This subsection will be focused on optical fiber sensors based on intensity detection. Even with a small number of works in this domain, compared to that of wavelength based sensors, there are some interesting publications to consider, such as the results reported with interferometers [40]-[43], 
TABLE I

REPORTED DNA HYBRIDIZATION SENSORS BASED ON WAVELENGTH SHIFT MEASUREMENTS

\begin{tabular}{|c|c|c|c|c|c|c|}
\hline Type & $\begin{array}{l}\text { Optical Fiber } \\
\text { Structure }\end{array}$ & $\begin{array}{l}\text { Sensitivity } \\
\mathrm{nm} / \mathrm{RIU} * \star\end{array}$ & Target & Detection Range & LOD & Ref. \\
\hline SPR & - & $\begin{array}{c}0.0035 \mathrm{~nm} \\
\text { per } \mathrm{pg} / \mathrm{mm}^{2}\end{array}$ & 5’-GGATAAACGTGCCССTTTTTTTTTT-3’ & $0.5-5 \mu \mathrm{M}$ & $\sim 0.5 \mu \mathrm{g} / \mathrm{ml}$ & [19] \\
\hline SPR & $400 \mu \mathrm{m}$ CRMOF & - & $\begin{array}{l}\text { Mycobacterium Avium Paratuberculosis andM. bovis } \\
\text { specific regions }\end{array}$ & $500-250 \mathrm{nM}$ & - & {$[20]$} \\
\hline SPR & $\begin{array}{l}\text { Graphene/Au } \\
\text { film/D-POF }\end{array}$ & 1227 & 5'-GTT TGA CAA ACA TCA AGA CAG AAG-3' & $100 \mathrm{pM}-1 \mu \mathrm{M}$ & 100 pM* & {$[21]$} \\
\hline LSPR & $\begin{array}{l}600 \mu \mathrm{m} \text { core fiber } \\
\text { tips }\end{array}$ & 218.98 & $\begin{array}{c}\text { 5’- } \\
\text { TTTTGGCTTTTGTGTGTCCCTTCCCTTCCCTGTGT } \\
\text { GTTTT-3’’ }\end{array}$ & $50-500 n M$ & $50 \mathrm{nM}^{*}$ & {$[22]$} \\
\hline LSPR & $\begin{array}{l}\text { 4/125 } \mu \mathrm{m} \\
\text { core/cladding SMF } \\
\text { tip. }\end{array}$ & $220-230$ & $\begin{array}{l}\text { 5'-CAACCGGTTATTTTTCTACA-3' } \\
\text { Analogue to } 16 \text { s rRNA subunit sequence of archaea }\end{array}$ & $100 \mu \mathrm{M}-1 \mathrm{fM}$ & $10 \mathrm{fM}$ & {$[23]$} \\
\hline LPG & Side-polished SMF & 909.1 & 5'-CCC CTT TTG TCG TTT TCA CCT CGC TG-3' & $1 \mu \mathrm{M}$ & $1 \mu \mathrm{M}^{*}$ & {$[24]$} \\
\hline LPG & SMF & 62 & 5'-GCGTGTGTTCGGTGCCCATTTCACC-3' & - & $62 \mathrm{nM}$ & {$[25]$} \\
\hline LPG & $\begin{array}{l}\text { High numerical } \\
\text { aperture SMF }\end{array}$ & $\begin{array}{l}160-250 \\
\mathrm{pm} / \mu \mathrm{M}\end{array}$ & 5'-GGC GAC TGA CTG TGC-3' & - & $10 \mathrm{nM}$ & {$[26]$} \\
\hline TFBG-SPR & - & - & - & $50-100 \mu \mathrm{M}$ & $50 \mu \mathrm{M}^{*}$ & [39] \\
\hline$\mu \mathrm{FBG}$ & $\begin{array}{l}\text { Tapered and } \\
\text { stretched MMF }\end{array}$ & 425 & 5’-CATCAATGTATCTTATCATGTCTGGA-3’ & $1 \mu \mathrm{M}$ & $1 \mu \mathrm{M} *$ & {$[27]$} \\
\hline$\mu \mathrm{FBG}$ & Tapered MMF & 215 & 5'-CA TCA ATG TAT CTT ATC ATG TCT GGA-3' & $0.1-1 \mu \mathrm{M}$ & $500 \mathrm{nM}^{*}$ & {$[28]$} \\
\hline Interferometry & Fabry Perot & - & $\begin{array}{l}\text { 5'-CATCAATGTATCTTATCATGTCTGGA-3' } \\
\text { Francisella tularensis specific sequence }\end{array}$ & $53-152 \mu \mathrm{M}$ & $53 \mu \mathrm{M}^{*}$ & [29] \\
\hline Interferometry & Microfiber/taper & $7.24 \mathrm{~nm} / \mu \mathrm{M}$ & 5' -CA TCA ATG TAT CTT ATC ATG TCT GGA -3' & $100 \mathrm{pM}-1 \mu \mathrm{M}$ & $100 \mathrm{pM}$ & {$[30]$} \\
\hline Interferometry & Microfiber /taper & $\begin{array}{c}1676 \\
0.93 \mathrm{~nm} / \mu \mathrm{M}\end{array}$ & 5’-CA TCA ATG TAT CTT ATC ATG TCT GGA-3' & - & $4.84 \mathrm{pM}$ & [31] \\
\hline Interferometry & Microfiber/ Sagnac & 13488 & 5'-CATCAATGTATCTTATCATGTCTGGA-3' & $100 \mathrm{pM}-1 \mu \mathrm{M}$ & 75 pM & {$[32]$} \\
\hline $\begin{array}{l}\text { Taper/interfero } \\
\text { meter }\end{array}$ & $\begin{array}{l}\text { SMF of } 125 \mu \mathrm{m} \\
\text { diameter/ taper }\end{array}$ & $\begin{array}{l}1905.7 \\
1.2862 \\
\mathrm{~nm} / \mathrm{nM}\end{array}$ & $\begin{array}{c}\text { 5'-CTT CGG ATT GTA AAG TTC ADT-3' } \\
\text { Leptospira }\end{array}$ & $0.1-1 \mathrm{nM}$ & $0.1 \mathrm{fM}$ & {$[33]$} \\
\hline Interferometry & Microfiber/taper & - & $\begin{array}{c}\text { 5'-TCA AGA CTC TGT TCT GAA CGT ACG GT-3' } \\
\text { 5'-CATCAATGTATCTTATCATGTCTGGA-3' }\end{array}$ & $1 \mathrm{pM}-1 \mu \mathrm{M}$ & $10 \mathrm{pM}$ & {$[34]$} \\
\hline Interferometry & $\begin{array}{c}\text { Single-mode } \\
\text { nonadiabatic/taper }\end{array}$ & - & 5'-GGCAGAGGCATCTTCAACGATGGCC-3' & $0.2-1 \mu \mathrm{M}$ & $200 \mathrm{nM}$ & {$[35]$} \\
\hline
\end{tabular}

*It is not a calculated LOD but lowest detected concentration.

** Sensitivity is in nm/RIU unless otherwise stated

fluorescence based sensors [43]-[45], and TFBGs [45].

Microfibers have also been used for intensity measurements. Particularly, a compact square-microfiber integrated biosensor was proposed and experimentally demonstrated for label-free detection of ssDNA in [40]. The proposed biosensor was constructed by splicing a square microfiber fabricated using a square fiber based on flame scanning technique with two segments of standard SMFs. The square microfiber with strong evanescent field is functionalized with a monolayer of poly-L-lysine. The functionalization allows the immobilization of thebiorecognition ssDNA probe to capture the cssDNA target. The transmission spectral response of the obtained biosensor was monitored during the ssDNA-cssDNA hybridization process in real time. The minimum concentration of cssDNA detected by this sensor was $10 \mathrm{nM}$ [40]. Here, it is important to note that the LOD value (10 nM) obtained in this case is several orders of magnitude higher than the LOD reported by the wavelength based microfiber sensors, which is in the order of pM [31], [32], [34], as described in the previous subsection.

In addition, a thin-core-fiber (TCF) based dual S-tapered fiber sensor for label free ssDNA-cssDNA hybridization detection has been proposed and experimentally demonstrated in [41]. The biosensor was developed by splicing a TCF segment of about $645 \mu \mathrm{m}$ in length with two segments of SMF. Two S-tapers were fabricated at the splicing joints by using a commercial fusion splicer (see Fig. 2). Label-free detection of cssDNA was achieved by functionalizing the fiber surface with a monolayer of poly-L-lysine and ssDNA probes.

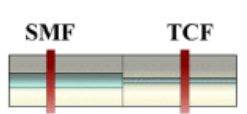

(a)

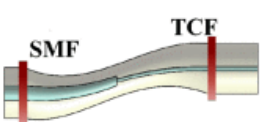

(b)

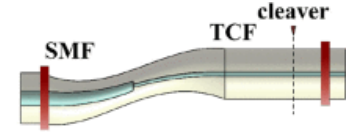

(c)
Fig. 2. Schematic illustration of the fabrication procedure for the TCFbased interferometer. (a) splicing of the TCF with the lead-in SMF (b) fabrication of the S-taper at the splicing joint (c) cutting the free end of the TCF using a fiber cleaver. Reprinted with permission from [41]. Copyright $@$ [2019] IEEE .

The sensor functionalization and the hybridization processes for $1 \mathrm{nM}$ ssDNA were successfully monitored in real-time.

Optical fiber interferometers have also relevance in the detection of DNA hybridization process based on intensity. A quite new interferometer design, known as specklegram interferometer, has been proposed for the detection of ssDNA [42]. The base of this new design is a large-core optical fiber that can support hundreds of eigenmodes. The interference pattern of the different eigenmodes propagating along the fiber forms a fiber specklegram [42]. Thus, the large-core fiber can 
be considered as an interferometer itself. A specklegram is determined by the relative amplitude and phase of the individual eigenmodes. The specklegram interferometer presents the lowest detected concentration of ssDNA hybridization in the domain of intensity based optical fiber sensors. This compact and ultrasensitive sensor based on fiber specklegram was functionalized with a monolayer of poly-Llysine and achieved a detection of $0.1 \mathrm{fM}$ ssDNA concentration [42]. Moreover, the well-known temperature cross-sensitivity issue in many optical fiber devices has been entirely removed in this biosensor by the introduction of a reference optical fiber channel along with the rest of microfluidic channels of the device.

Among the techniques used for ssDNA detection, fluorescence has attracted particular attention because it provides fast, accurate, sensitive and selective platforms [43]. Hence, a novel enzyme-enhanced fluorescence detection using etched optical fiber sensor has been developed for the detection of ssDNA in [43]. In this case, the hybridization process occurs on the etched optical fiber, and the double strand DNA was then bound with streptavidin labelled horseradish peroxidase (streptavidin-HRP). The cssDNA target was quantified through the fluorescence detection of the compound generated under the catalysis of HRP. The sensor reported a linear behavior in the range from 1.69 to $169 \mathrm{pM}$ concentrations of ssDNA achieving a LOD of $1 \mathrm{pM}$.

Fluorescence mediated detection has also been applied for the detection of microorganisms. A novel portable evanescent wave fiber biosensor was developed for the labelled detection of Shigella [44]. Shigella is a genus of bacteria that causes Shigellosis, a gastrointestinal infection in humans. Traditional analytical methods for Shigella detection include bacterial cultivation, serological methods, and PCR. In the experiments with the fluorescence fiber optic biosensor for Shigella, the sensing time, sensitivity, specificity, and reusability of the biosensor were validated. The LOD of the portable fiber-optic biosensor was $0.1 \mathrm{nM}$ of Shigella, similar to that of real-time PCR. Hence, the biosensor was advantageous over the traditional detection methods in terms of speed, simplicity, and suitability for on-site detection.

A specific sequence of Hepatitis A virus was presented as another microbial target for optical fiber sensors in [46]. Hepatitis A virus infection is responsible for around half of the total number of hepatitis infections diagnosed worldwide. In this case, a chemiluminescent fiber optic sensor was developed for the detection of RNA sequence and its complementary ssDNA. The sensor showed a linear detection range between $5 \mathrm{pg} / \mu \mathrm{l}$ to $10 \mathrm{ng} / \mu \mathrm{l}$ for ssDNA $\left(\mathrm{R}^{2}=0.93\right)$ and achieved a LOD of $5 \mathrm{pg} / \mu \mathrm{l}$ for a ssDNA specific sequence.

Finally, a device with potential to act as a thermo-cycling device with high multiplexing capabilities for genomic amplification in PCR was reported in [45]. PCR is a widely used diagnosis method, which typically require series of 20-40 repeated temperature changes. The proposed device consists of a TFBG based hot-wire sensor where no external heat source or temperature probe is required. It is also the first combination of internal temperature probing and fiber heating together with fluorescence imaging. This combination allowed the miniaturization of a microarray like device and eliminated the need for external thermal cycling. The sensor was successfully tested with a solution of $0.1 \mu \mathrm{M}$ fluorescent cssDNA target. The main characteristics of the previously reported works are summarized in Table 2, together with the details of the ssDNA target.

MBs, and DNA origami structures. These three synthetic

TABLE II

REPORTED DNA HYBRIDIZATION SENSORS BASED ON INTENSITY MEASUREMENTS

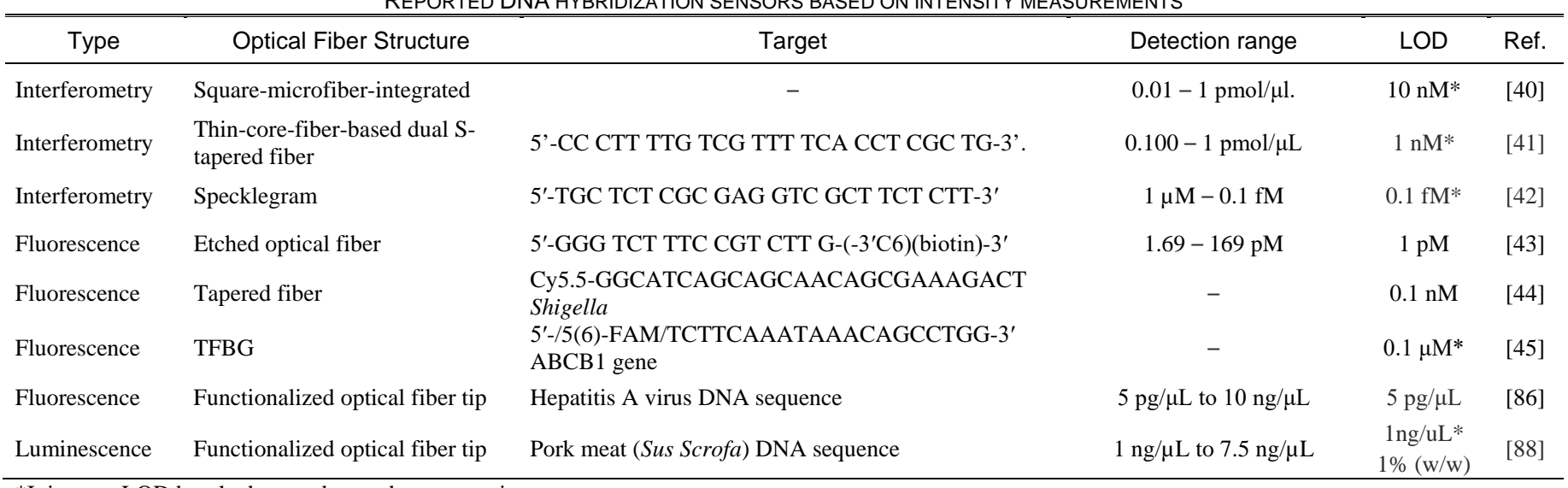

*It is not a LOD but the lowest detected concentration.

\section{SYNTHETIC SSDNA FOR SSDNA TARGETS.}

Most of the optical fiber sensors composed of DNA sequences are based on ssDNA-cssDNA hybridization as described in the previous section. The use of synthetic nucleic acids confers several advantages to nucleic acids structures that becomes them good alternatives for the detection of ssDNA. According to this, new strategies have been developed in optical fiber sensing thanks to the use of PNAs, structures are described in this section. Furthermore, the most relevant approaches based on the combination of such structures with optical fibers are also presented.

PNAs are synthetic DNA analogues that have been employed in biosensing for the detection of ssDNA short sequences, although the use of PNA in fiber optical sensors is not widely extended. A PNA is a synthetic nucleic acid analogue whose deoxyribose phosphate backbone is replaced by a pseudo-peptide polymer to which the nucleobases are 
linked. PNAs hybridize with cssDNA with remarkably high affinity and specificity, essentially because of their uncharged and flexible polyamide backbone [14]. In addition, PNA exhibits chemical and thermal stability in conditions where DNA would undergo degradation. PNAs are insensitive to ionic strength and $\mathrm{pH}$ changes and are resistant to enzymatic cleavage inside living cells. A single mismatch in PNA/DNA heteroduplexes decreases the melting temperature more than in DNA/DNA duplexes (about $15{ }^{\circ} \mathrm{C}$ compared with $4{ }^{\circ} \mathrm{C}$ ) thus demonstrating the higher level of selectivity of PNA compared with DNA [47].

In the literature, few papers describe the use of PNA for the detection of cssDNA with optical fiber sensors [48]-[52]. The first work consisted of using PNAs on a microstructured optical fiber [48]. After a functionalization of the silica hole surfaces, which allows the immobilization of PNA, they detected the selective binding with full-complementary ssDNA sequences. Experimental results confirmed the specificity of the probe that was able to discriminate the ssDNA with only a single nucleotide variation. The same research group, Sozzi et al, also investigated a biosensor based on LPG with PNA for the detection of cssDNA [49]. A wavelength shift of $1.2 \mathrm{~nm}$ for a $120 \mathrm{nM}$ cssDNA solution was measured in this case.

Later, a novel sensing approach based on a functionalized microstructured optical fiber-Bragg grating for specific cssDNA sequences detection was described [50]. Here, PNAs were used as probes for the detection through hybridization of a single nucleotide DNA mutation implicated in cystic fibrosis disease. The sensor was able to detect $100 \mathrm{nM}$ solution of cssDNA with the specific mutation. The wild-type sequence was also tested to demonstrate the specificity of the sensor.

In addition, a sensor based on optical fiber ring cavity utilizing a double TFBG as detector element was employed for ssDNA detection [51]. The sensor functionalized with PNA probes could discriminate single nucleotide variations and allowed the detection of $10 \mathrm{nM}$ cssDNA solution. Recently, an optical fiber sensor based on a Bragg grating inscribed on microstructured optical fibers was developed. The microchannels of the microstructured fiber were internally functionalized with an specific PNA probe for a gene tract of the genetically modified Roundup Ready soy [52]. The fiber channels permitted to achieve a label free detection of around $30 \mathrm{ng} / \mathrm{ml}$ concentrations of cssDNA using volumes as low as $100 \mu \mathrm{l}$.

MBs are single-stranded oligonucleotide hybridization probes that were initially developed by Tyagi and Kramer in 1995 [53]. These probes consist of a stem-and-loop structure depicted in Fig. 3. The loop contains a probe sequence complementary to a target sequence, whereas the stem part is formed by the annealing of complementary arm sequences located on either extremes of the probe sequence. Their unique structure can provide two well-differenced conformations: an open or extended conformation, and a closed conformation commonly named as hairpin. The principle of operation of MBs consists of the fact that the hairpin stem formed by the complementary sequences cannot coexist with the rigid double chain that is formed when the probe hybridizes with the target sequence. As a result, the MBs undergo a conformational change that forces to separate the arm sequences.

This conformational change can be monitored optically if the arm sequences are properly modified at both ends using a fluorophore and a quencher respectively. This way, on one hand the fluorescent emission from the fluorophore is neutralized by the quencher before the conformational change (see Fig. 3a). On the other hand, once the probe hybridizes with its target, fluorophore and quencher molecules are far enough and, therefore, the fluorescence can be appreciated and
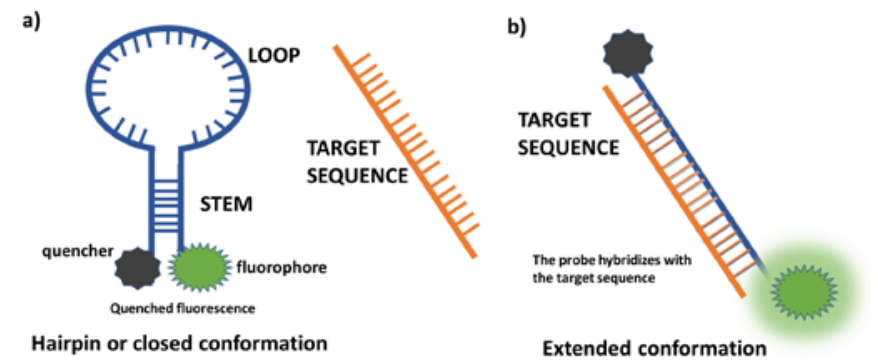

Fig. 3. (a) $M B$ in a hairpin conformation with the stem-and-loop structure with the quencher and the fluorophore linked at the end of the arm sequences, and, separately, a target sequence; (b) MB in extended conformation, where the target sequence hybridizes with the probe sequence. Quencher and fluorophore are distanced enough, and fluorescence emission can be detected.and measured.

detected (Fig. 3b).

MB-based sensing systems are expanding rapidly and provide a promising bioanalytical tool for cost-efficient and reliable analysis for research and biomedical diagnostics [15]. Thus, optical fiber structures can be used as interesting sensing platforms to locate MBs and obtain powerful and selective DNA based detection systems. The first important work that developed this new strategy was reported by Liu et al. [54]. There it was proposed an evanescent wave based sensor that detected non-labeled DNA target in real time with high sensitivity and one-base-mismatch selectivity. This work opened the door to other more recent approaches with microstructured optical fibers [55], [56].

One of the last relevant advances in this field has been reported by Giannetti et al [55]. In this work, a tapered tip of an optical fiber was coupled with MBs for the detection of DNA. The tip was fabricated by a dynamic etching method, rotating and dipping an optical fiber in an aqueous hydrofluoric acid solution and controlling precisely different parameters, thus obtaining short taper length $(\sim 200 \mu \mathrm{m})$, large cone angle (from $15^{\circ}$ to $40^{\circ}$ ), small probe tip dimension (less than $30 \mathrm{~nm}$ ), and roughness below $10 \mathrm{~nm}$. Then, the created nanotip was functionalized with amino group by a silanization process.

Afterwards, N-succinimidyl 3-(2-pyridyldithio) propionate was used as a crosslinker between these amino groups available onto the nanotip and the - $\mathrm{SH}$ groups of the linear probe of the MB. The performance of the fiber nanotip to act as sensor was evaluated using a $635 \mathrm{~nm}$ laser diode as a light source. The resultant spectra as well as the fluorescence signal emitted by the fluorophore (ATTO647n) were recorded as a function of time when exposed to different concentrations of the target. The results are shown in Fig. 4, and a LOD of 0.57 $\mathrm{nM}$ was achieved. 


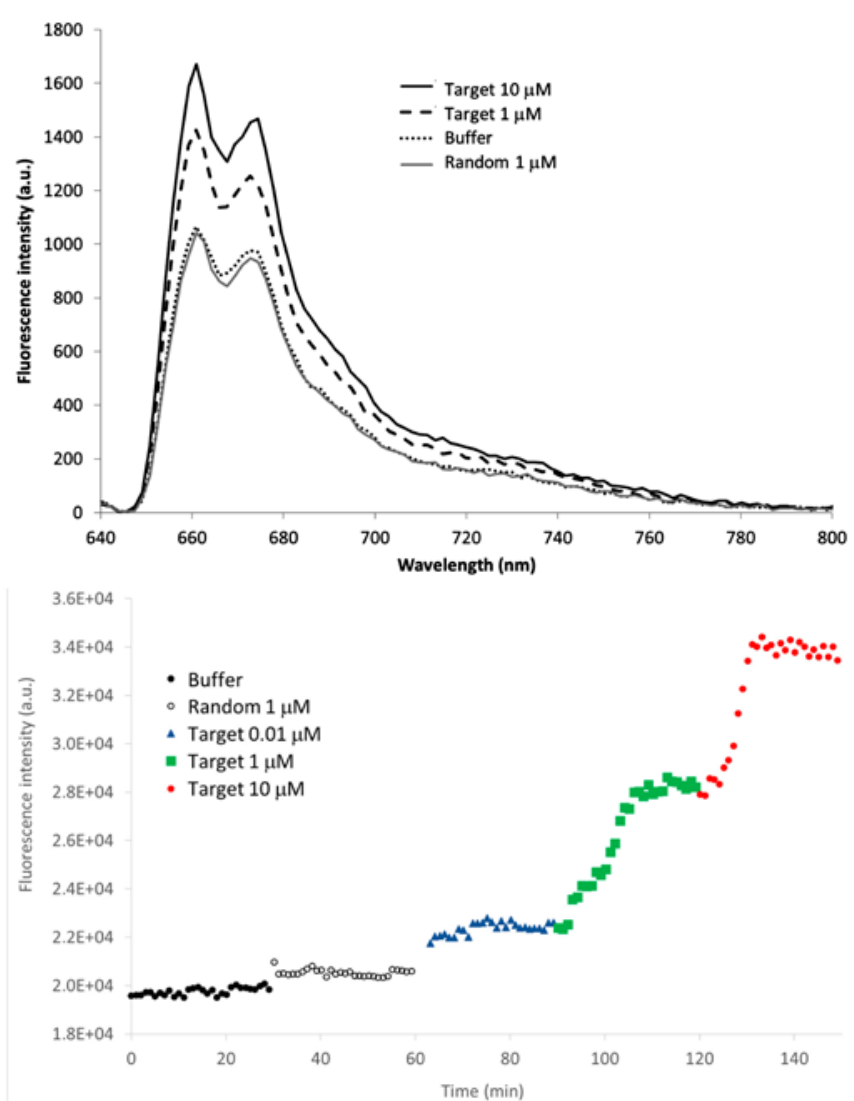

Fig. 4. (a) Spectra from the nanotip optical fiber with the $M B$ in presence of the target $(1 \mu \mathrm{M}$ and $10 \mu \mathrm{M})$ and of a random sequence $(1 \mu \mathrm{M})$. $\lambda$ ex $635 \mathrm{~nm}$ and exposure time $1 \mathrm{~s}$. (b) Fluorescence signal along the time during the interaction of the MB immobilized onto the nanotip with a random sequence, and the target at different concentrations $(0.01 \mu \mathrm{M}, 1 \mu \mathrm{M}$ and $10 \mu \mathrm{M})$. Reprinted with permission from [55]. Creative Commons Attribution license (http://creativecommons.org/licenses/by/4.0/).

Other interesting approach with MBs onto suspended core optical fibers was reported by Nguyen et al. [56], following the scheme presented in Fig. 5. The inner surface of the suspended core fiber was first coated with polymeric multilayer films by the layer-by-layer nanoassembly technique. The final multilayer provides amino groups for immobilization of biotinylated MB through a biotinstreptavidin-biotinylated MB link.

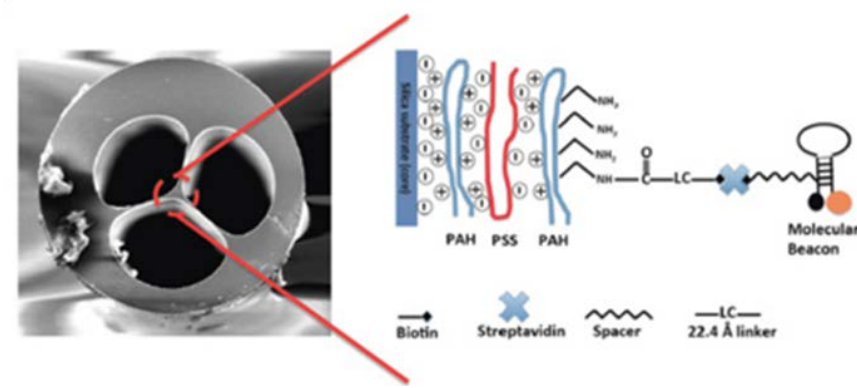

Fig. 5. SEM image of the suspended core fiber used in [56], and the schematic diagram of the final surface state of the MB functionalized fiber. Reprinted with permission from [56]. (CThe Optical Society.

The reflection configuration setup consisted of a laser and a spectrometer. After the hybridization test, MB-immobilized suspended core fiber was loaded with different DNA solutions. Fluorescence was recorded showing the capability of the proposed sensor to detect complementary DNA sequences while discriminating sequences differing from the target by just one base.

DNA origami is a particular technique which permits to design arbitrarily shaped complex three dimensional nanostructures. More specifically, DNA origami is a process of molecular self-folding: a long ssDNA (scaffold), is folded into prescribed objects by hundreds of short synthetic DNA oligonucleotides, which are designed to be complementary to different parts of the scaffold DNA [16]. In the last years, not only the applications of DNA origami have emerged in nanofabrication [57], [58] or nanomechanics [59] fields, but also in drug delivery [60], or biosensing [61]. Nevertheless, the strategies to immobilize DNA origami into optical fiber surfaces are in an incipient stage, with very few publications [17], [18].

Torelli et al. [62] have reported a fiber optic sensor based on DNA origami nanorobot to detect tobacco mosaic virus (TMV). The nanorobot was designed and constructed by folding DNA-based scaffold strands and DNA-based staple strands until the formation of a $3 \mathrm{D}$ origami nanostructures with a flap. These nanorobots were immobilized on the surface of the end facet of an optical fiber using an ssDNA capture probe composed of a capture sequence and an anchor sequence. The DNA origami-optical fiber sensor was immersed in a solution containing TMV target sequences enabling molecular recognition and hybridization, and then the test probe was dipped into a luminol-based solution for evaluation of the presence of the target in the test sample. The well-folded DNAzyme catalyzes the $\mathrm{H}_{2} \mathrm{O}_{2}$-mediated oxidation of luminol to yield chemiluminescence. The light emitted was collected by the optical fiber and monitored.

In a different approach, Daems et al. [63] immobilized 3D DNA origami structures onto optical fibers in order to develop fiber optic SPR sensors with nanoscale precision. First, two gold sputtered coated optical fibers were functionalized with thiol-modified ssDNA sequences as hybridization probes for binding the DNA origami structures. In addition, two distinct DNA origami structures (LS and DE types) were designed to position thrombin specific aptamers with different densities and distances from the SPR surface of the fibers. A third optical fiber was functionalized with tetrahedrons as carriers of the thrombin bioreceptors and was used as a reference. An overview of the overall schematic is shown in Fig. 6. The developed sensors showed an increased detection range for the DNA origami compared to the tetrahedron-based biosensors. However, this field related with DNA origami structures combined with optical fibers is being initially explored, and the advent of further promising research is required.

\section{SSDNA RECEPTORS FOR NON-DNA TARGETS}

Most of the optical fiber sensors composed of DNA sequences are based on DNA hybridization, as described in the previous section. Many of such sensors have been reported demonstrating the detection of a complementary DNA strand using different optical methods, structures or configurations. Nevertheless, there also exist other optical fiber DNA based sensors, which permit to develop sensing applications for a 


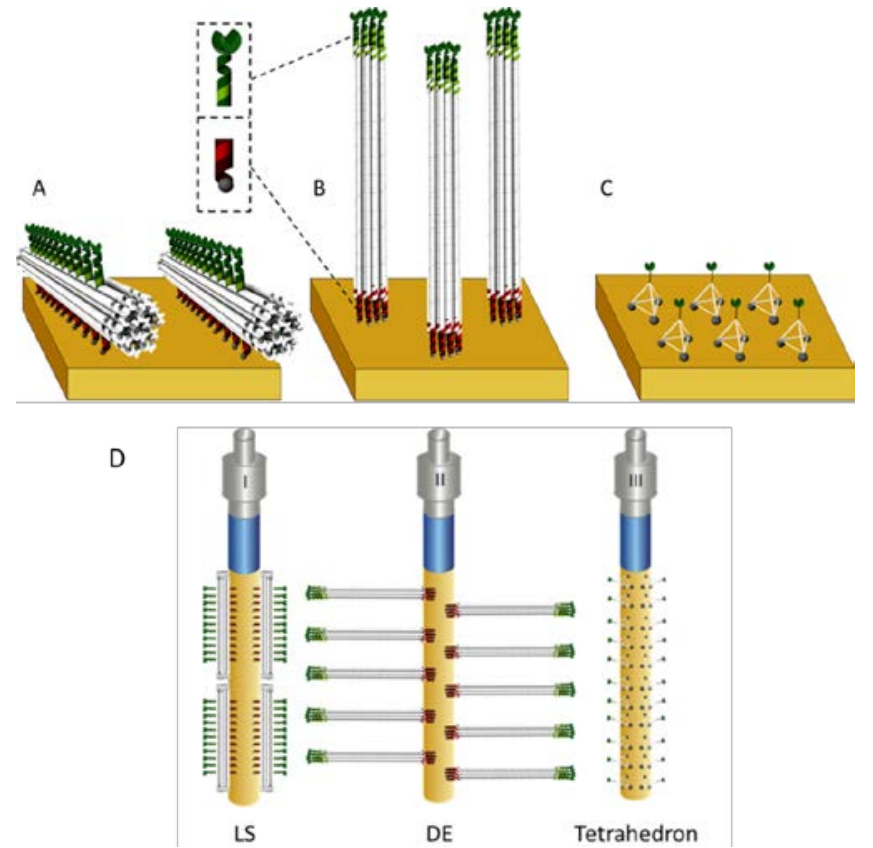

Fig. 6. Schematic of bioreceptor patterning onto a gold surface using: (a) 3D DNA LS origami, (b) 3D DNA DE origami, and (c) 3D DNA tetrahedron, containing bioreceptors (dark green) and thiol groups (dark gray spheres) for surface attachment. (d) Functionalization of the three fiber optic-SPR biosensors with DNA nanostructures Zoomin: representation of the distinct set of SsDNA (light green) for specific attachment of the bioreceptors (dark green) to the DNA origami and the distinct set of ssDNA (light red) for specific attachment of the DNA origami structures to the biosensing surface (dark red). Readapted with permission from [63]. Copyright (c) (2018) American Chemical Society.

widely range of biomolecules such as adenosine [64], dopamine [65], nicotine [66], cancer biomarkers [67], other enzymes [68], RNA sequences [69], [70]; other compounds as heavy metal ions [71], [72]; or even physical magnitudes, such as temperature [73].

All these sensors can be also classified into different types depending on their sensing methods as in previous section. Within the intensity based sensors, most of the studies recently presented are chemiluminescent or fluorescence based sensors by means of the functionalization of the end facet of optical fibers [46], [74]-[76] or evanescent wave sensors [64], [77]. Among the wavelength based sensors, some interferometers [66], [78], lossy mode resonance based devices [79], [80] and SPR based devices [81]-[85] can be found in literature. Regarding the latter ones, there are some relevant works where interesting approaches which have been developed thanks to the use of TFBGs in combination with the SPR phenomenon in order to obtain better performances. Here, Albert et al. [82] designed and implemented high-resolution fiber optic aptasensors, demonstrating an order of magnitude improvement in the determination of the SPR shifts under biochemical interactions at the fiber surface. The proposed sensing structure was tested with thrombin aptamers, obtaining a LOD of 22nM. Other relevant optical structure based on TFBG coated by Au film and a graphene thin-film was reported by $\mathrm{Hu}$ et al. [83]. The resultant device allowed the detection of dopamine molecules, showing a linear response in the range from $10^{-13} \mathrm{M}$ to $10^{-8} \mathrm{M}$ with an LOD of $1.6 \times 10^{-13} \mathrm{M}$. Alternative approaches have been also developed aptasensors based on lossy mode resonances (LMRs) for specific purposes such as thrombin [80] or Creactive protein detection [79].

Among the most promising applications within the DNAbased optical fiber sensors domain, RNA and miRNA detection are especially relevant. Recently, it has been developed a fiber optic device to detect RNA sequences of Hepatitis A virus thanks to a sandwich type hybridization system [86]. In another relevant work, Qian et al. have reported [70] a high sensitivity microRNA detection technique using phenylboronic acid functionalized $\mathrm{Au}$ nanoparticles (PBA-AuNPs) in a fiber-optic SPR sensor. The miRNA sequence target in this work was Let-7a, a promising miRNA for cancer therapy. The inherent difficulty of detecting directly the hybridized RNA on the sensing surface was overcome thanks to the presence of PBA-AuNPs, which enabled the signal amplification of the miRNA target. Fig. 7 represents the miRNA detection process. First, the sensing surface is modified with the DNA capture and 6-mercapto-1-hexanol $(\mathrm{MCH})$. In a second stage, the RNA complementary is hybridized. In a final stage, the functionalized Au NPs bind selectively with the RNA, thus amplifying the signal. This method demonstrated a high selectivity when mismatched or random DNA and RNA sequences were also applied to the system. The miRNA detection offered a low LOD of 0.27 pM.

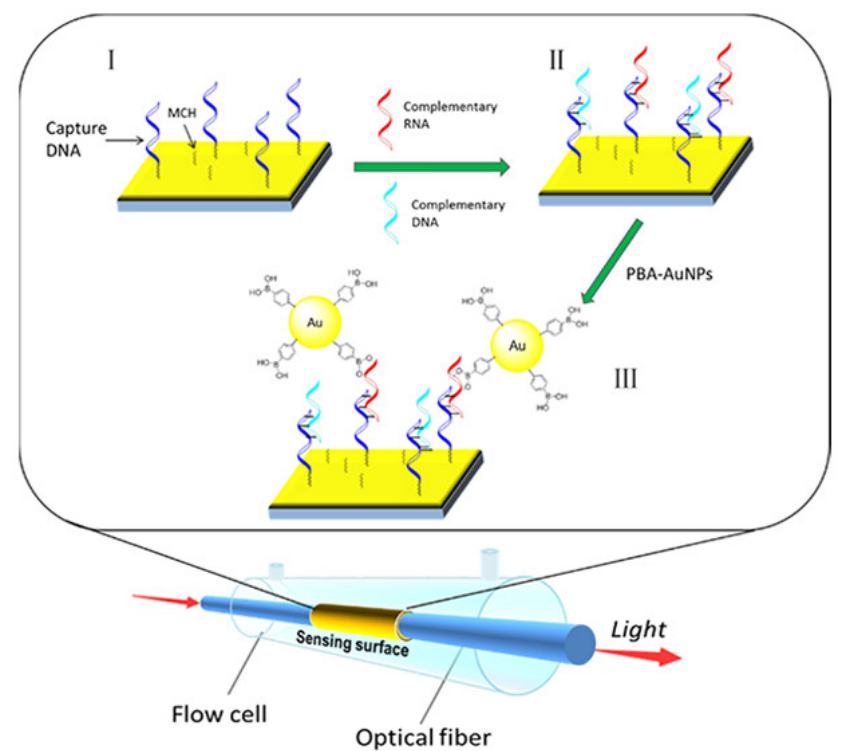

Fig. 7. Schematic representation of miRNA detection by fiber-optic SPR sensing system: (I) Capture DNA/MCH modification on the sensing surface. (II) Single-stranded RNA or DNA hybrid on the sensing surface. (III) PBA-AuNPs selectively bind with RNA to amplify the signal. Reprinted with permission from [70]. Copyright (c) (2018) American Chemical Society.

Not only DNA based coatings are proposed for biomolecule detection, but also can be implemented to measure physical magnitudes such as temperature. Thus, Hong et al. [78] proposed an interferometer based on a single mode-coreless silica-SMF structure. In this work, the coreless fiber segment was coated by a temperature sensitive coating composed of a DNA-cetyl tri-methyl ammonium (DNA-CTMA) thin solid film. The DNA-based thin film has a large negative thermooptical coefficient of $-3.4 \times 10^{-4 \circ} \mathrm{C}^{-1}$ in the temperature range from $20^{\circ} \mathrm{C}$ to $70^{\circ} \mathrm{C}$ without any observable thermal hysteresis. 
As a result, the temperature variations change the refractive index of the thin films, thus provoking a spectral shift of the fringes in the output interferometric signal. Some
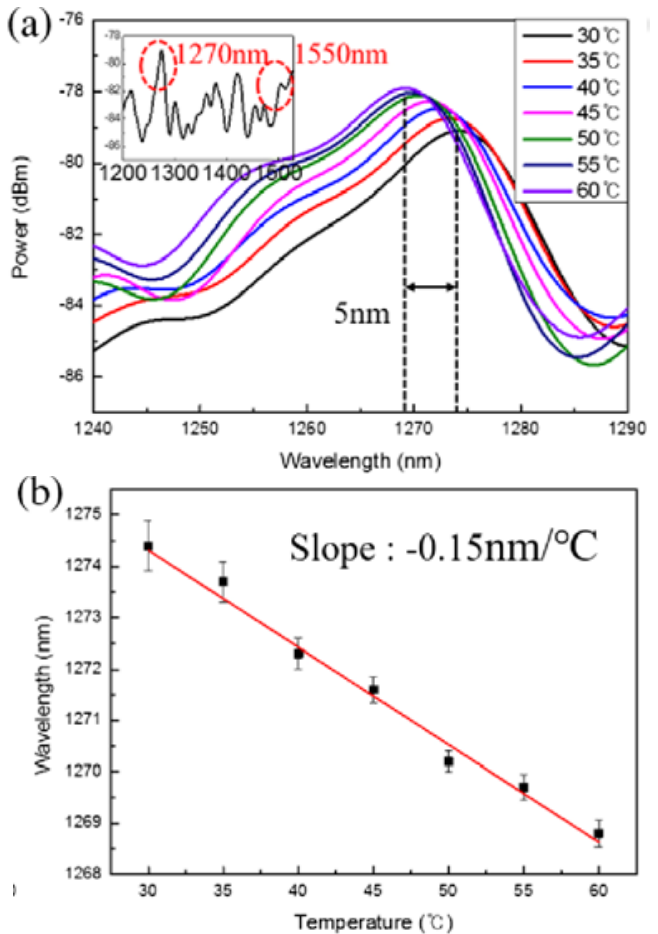

Fig. 8. Experimental measurements of the temperature sensor: (a) Spectral shifts between $30^{\circ} \mathrm{C}$ and $60^{\circ} \mathrm{C}$, (b) temperature dependence of peak wavelength. Reprinted with permission from [78]. (c) The Optical Society.

experimental results of this work are represented in Fig. 8, where it is possible to appreciate the large temperature range and the linear response with a sensitivity of $-0.15 \mathrm{~nm}{ }^{\circ} \mathrm{C}$.
Apart from the mentioned applications, mercury ions detection in solutions is currently another hot topic research. Recently, two different approaches have been proposed by means of fiber optic DNA based- devices [85], [87]. On one hand, Jia et al. designed an LSPR sensor with gold nanospheres on the end facet of a fiber for $\mathrm{Hg}^{2+}$ detection based on thymine- $\mathrm{Hg}^{2+}$-thymine base pair mismatches and the coupled plasmonic resonance effect [85]. This sensor offered a linear detecting range of $\mathrm{Hg}^{2+}$ from 1 to $50 \mathrm{nM}$ with a LOD of $0.7 \mathrm{nM}$. On the other hand, Polley et al. [87] presented a DNA biomaterial based fiber optic sensor with another $\mathrm{Hg}^{2+}$ detection strategy. In this work, optical fiber tips were coated with silver nanoparticles and then impregnated with a genomic DNA-dye-lipid complex with specific affinity to mercury ions. The sensing mechanism is based on the combination of the fluorescence with the SPR associated to the silver NPs. The specific detection of mercury ions at the nanomolar scale with few seconds reaction time was successfully demonstrated in this work. A summary of the presented works as well as other optical fiber DNA-based sensors recently reported is included in Table 3.

\section{DISCUSSION AND OUTLOOK}

This review includes many optical configurations and even when a similar optical configuration is used, there are several variables which make difficult to compare the sensors performance. Variations on the optical phenomenon or structure, the measurement setup, the functionalization of the surface, the receptor or the target affect to the results of the optical devices. The receptor varies, not only between those contributions, which are based on natural ssDNA and synthetic nucleic acids structures, but also inside those groups.

TABLE III

REPORTED SENSORS BASED ON SSDNA RECEPTORS FOR NON-DNA TARGET DETECTION

\begin{tabular}{|c|c|c|c|c|c|}
\hline Type & Structure and coatings & Target & Detection Range & LOD & Ref. \\
\hline LSPR & Au NPs based coating on fiber end facet & $\mathrm{Hg}^{2+}$ ions & $1 \mathrm{nM}-50 \mathrm{nM}$ & $0.7 \mathrm{nM}$ & [85] \\
\hline SPR on LPFG & $\mathrm{Au}$ - thiolated aptamer & microcystin-LR toxin & $5 \mathrm{nM}-300 \mathrm{nM}$ & $5 \mathrm{nM}$ & [90] \\
\hline SPR on TFBG & Fiber probe with Au coating + graphene film + aptamer & Dopamine in human serum & $0.1 \mathrm{pM}-10 \mathrm{nM}$ & $0.16 \mathrm{pM}$ & [65] \\
\hline SPR on TFBG & Fiber probe with Au film + aptamers & Thrombin & $0.1 \mu \mathrm{M}-5 \mu \mathrm{M}$ & $22 \mathrm{nM}$ & [82] \\
\hline SPR Intensity & Au film on cladding removed MMF & RNA sequence (Let-7a) & $0.27 \mathrm{pM}-100 \mathrm{nM}$ & $0.27 \mathrm{pM}$ & [70] \\
\hline Fluorescence & $\begin{array}{l}\text { Sandwiched type assembly with a fluorophore on etched } \\
\text { SMF fiber }\end{array}$ & Adenosine & $50 \mu \mathrm{M}-3.5 \mathrm{mM}$ & $25 \mu \mathrm{M}$ & [64] \\
\hline Fluorescence & Enhanced SPR with a dye and Ag NPs on optical fiber tips & $\mathrm{Hg}^{2+}$ ions & $9 \mathrm{nM}-50 \mu \mathrm{M}$ & $9 \mathrm{nM} *$ & [87] \\
\hline Fluorescence & $\begin{array}{l}\text { Evanescent wave of a SMF-MMF coated with a } \\
\text { fluorescence-labeled BPA-aptamer }\end{array}$ & Bisphenol A & $2-100 \mathrm{nM}$ & $1.86 \mathrm{nM}$ & [77] \\
\hline Fluorescence & Functionalized optical fiber tip & $\begin{array}{l}\text { Hepatitis A virus (RNA } \\
\text { sequence) }\end{array}$ & $50 \mathrm{pg} / \mu \mathrm{L}$ to $10 \mathrm{ng} / \mu \mathrm{L}$ & $50 \mathrm{pg} / \mu \mathrm{L}^{*}$ & {$[86]$} \\
\hline Fluorescence & $\begin{array}{l}\text { Etched and functionalized tip fibers coated with a } \\
\text { fluorophore }\end{array}$ & $\begin{array}{l}\text { reactive oxygen species } \\
\text { (ROS) generator: } \mathrm{Mn} 304 \\
\text { NPs }\end{array}$ & $0.13 \mu \mathrm{M}-1.36 \mu \mathrm{M}$ & $0.13 \mu \mathrm{M}^{*}$ & [89] \\
\hline Interferometer & $\begin{array}{l}\text { SMF-CSF-SMF interferometer coated with DNA and Cetyl } \\
\text { tri-methyl ammonium }\end{array}$ & $\begin{array}{l}\text { Temperature, Sensitivity of } \\
0.15 \mathrm{~nm} /{ }^{\circ} \mathrm{C}\end{array}$ & $20^{\circ} \mathrm{C}-70^{\circ} \mathrm{C}$ & -- & [78] \\
\hline Interferometer & Functionalized non-adiabatic tapered optical fiber & Nicotine & $10 \mu \mathrm{M}-100 \mu \mathrm{M}$ & $10 \mu \mathrm{M}^{*}$ & [66] \\
\hline
\end{tabular}


Different length and weight of the ssDNA sequences may also lead to different results.

However, some general conclusions can be extracted. In the field of fiber optic sensors, wavelength based configurations are more robust than intensity based techniques, which can be one of the main reasons for a larger number of contributions in the literature regarding DNA fiber optic sensor for the former. However, both configurations have permitted to obtain good results in terms of LOD.

Regarding wavelength based contributions to detect ssDNA-cssDNA hybridization, the optical sensors which achieved the best LOD are those based on LSPR and interferometers. In particular, LSPR biosensor based on arrays of metallic nanostructures on the end facets of optical fibers permitted to obtain an LOD of $10 \mathrm{fM}$ for ssDNA [23]. Concerning interferometers, a tapered optical fiber sensor used to detect a specific ssDNA sequence of Leptospira bacteria led to an LOD of $0.1 \mathrm{fM}$ for ssDNA [33]. In addition, the implementation of microfiber tapered interferometers has also led to good LOD results. A microfiber based sensor with a graphene oxide coating deposited around the silica microfiber reached an LOD of $4.84 \mathrm{pM}$ for ssDNA specific sequence solution [31] and a sensor based on a microfiber in reflection configuration functionalized with a monolayer of poly-Llysine achieved a minimum detectable concentration of $10 \mathrm{pM}$ for ssDNA [34].

Among the several techniques used for ssDNA detection using optical fiber sensors based on intensity, fluorescence and the interferometers based sensors are the most frequently used. Within the latter group, the specklegram interferometer presented the lowest detected concentration of ssDNA with $0.1 \mathrm{fM}$ ssDNA concentration detection [42], which equals the minimum LOD achieved with wavelength based optical fiber sensors [33]. On the other hand, with fluorescence based sensors the minimum LOD was achieved with a novel enzyme-enhanced fluorescence detection on etched optical fiber sensor, 1pM ssDNA [43].

PNAs are analogues of the DNA and allow the detection of ssDNA specific sequence in the same way as ssDNA receptors. They have been used in the field of biosensors, but at the moment just a few papers have been described about their implementation in fiber optic biosensors. Taking into account this point, we can see that the LOD obtained with the optical fiber sensors which used PNAs as receptors is acceptable. The minimum detected concentration by these biosensors is in the range from $10 \mathrm{nM}$ to $120 \mathrm{nM}$ of ssDNA. Therefore, PNAs are presented as a promising tool for the fabrication of sensitive and highly selective biosensors.

In a similar way, MBs and origami optical fiber sensors are in an incipient stage. Both techniques offer interesting approaches to detect multiple and selective biocompounds thanks to their customized designs, and could be a new trend in the near future

Regarding the LOD obtained for the detection of ssDNA using different receptors on optical fiber sensing platforms, i.e. sensors that employ ssDNA and synthetic nucleic acids receptors for the detection of different targets (non-DNA targets), the lowest LOD obtained with natural ssDNA short sequences as receptors for the detection of non-DNA targets has been reported for the detection of miRNA complementary sequence, with an SPR sensor that included phenylboronic acid functionalized $\mathrm{Au}$ nanoparticles with ssDNA short sequences on the surface. The LOD achieved by this sensor was $0.27 \mathrm{pM}$ of the complementary microRNA sequence [70]. However, sensors with synthetic nucleic acids receptors reported also low LODs for the detection of different molecules. The lowest LOD presented in this case was obtained by a nano-scale metalcoated TFBG imprinted in a commercial single mode fiber core. The receptors of this sensor were selective aptamers for dopamine molecule and this sensor reported an LOD of 0.16 pM for dopamine [65].

To conclude, it has been shown throughout this work the increasing interest in the use of DNA for sensing technologies. The detection of specific DNA sequences offers advantages for medical diagnosis, food industry and environmental analysis. Moreover, the development of synthetic nucleic acids receptors provides new tools for the sensing technology. In particular, synthetic nucleic acids are more stable than natural ones and they offer ample possibilities in the detection of a wide range of targets, including non-DNA targets, which can broaden the application scope of natural DNA receptors.

\section{REFERENCES}

[1] X. D. Wang and O. S. Wolfbeis, "Fiber-Optic Chemical Sensors and Biosensors (2013-2015),” Anal. Chem., vol. 88, no. 1, pp. 203-227, 2016.

[2] A. B. Socorro-Leránoz, D. Santano, I. Del Villar, and I. R. Matias, "Trends in the design of wavelength-based optical fibre biosensors (2008-2018),” Biosens. Bioelectron. X, vol. 1, no. April, p. 100015, 2019 .

[3] F. Chiavaioli, C. A. J. Gouveia, P. A. S. Jorge, and F. Baldini, "Towards a uniform metrological assessment of grating-based optical fiber sensors: From refractometers to biosensors," Biosensors. 2017

[4] C. Caucheteur, T. Guo, and J. Albert, "Review of plasmonic fiber optic biochemical sensors: improving the limit of detection,” Anal Bioanal Chem, 2015.

[5] X. D. Wang and O. S. Wolfbeis, "Fiber-Optic Chemical Sensors and Biosensors (2015-2019),” Anal. Chem., vol. 92, no. 1, pp. 397-430, 2020.

[6] S. Yin and P. Ruffin, "Fiber Optic Sensors,” Wiley Encycl. Biomed. Eng., vol. 84-2, p. 801, 2006.

[7] H. Šípová and J. Homola, "Surface plasmon resonance sensing of nucleic acids: A review,” Anal. Chim. Acta, vol. 773, pp. 9-23, 2013.

[8] M. Zourob, Recognition receptors in biosensors. 2010.

[9] C. R. Zamarreño, A. B. Socorro, P. Sanchez, I. R. Matias, and F. J. Arregui, Fiber-optic biosensors, Taylor and. CRC Press, 2015.

[10] X. D. Wang and O. S. Wolfbeis, "Fiber-optic chemical sensors and biosensors (2008-2012),” Anal. Chem., vol. 85, no. 2, pp. 487-508, 2013.

[11] A. Hanif, R. Farooq, M. U. Rehman, R. Khan, S. Majid, and M. A. Ganaie, “Aptamer based nanobiosensors: Promising healthcare devices,” Saudi Pharm. J., vol. 27, no. 3, pp. 312-319, 2019.

[12] V. Perumal and U. Hashim, “Advances in biosensors: Principle, architecture and applications,” J. Appl. Biomed., vol. 12, no. 1, pp. 1-15, 2014.

[13] H. Li, T. H. LaBean, and K. W. Leong, "Nucleic acid-based nanoengineering: Novel structures for biomedical applications,” Interface Focus, vol. 1, no. 5, pp. 702-724, 2011.

[14] F. Pellestor and P. Paulasova, "The peptide nucleic acids (PNAs), powerful tools for molecular genetics and cytogenetics,” Eur. J. Hum. Genet., vol. 12, no. 9, pp. 694-700, 2004.

[15] M. Stobiecka and A. Chałupa, "Biosensors based on molecular beacons,” Chem. Pap., vol. 69, no. 1, pp. 62-76, 2015.

[16] P. Wang, T. A. Meyer, V. Pan, P. K. Dutta, and Y. Ke, "The Beauty and Utility of DNA Origami,” Chem, vol. 2, no. 3, pp. 359-382, 
Mar. 2017

[17] D. Daems, W. Pfeifer, I. Rutten, B. Saccà, D. Spasic, and J. Lammertyn, "Three-Dimensional DNA Origami as Programmable Anchoring Points for Bioreceptors in Fiber Optic Surface Plasmon Resonance Biosensing,” ACS Appl. Mater. Interfaces, vol. 10, no. 28, pp. 23539-23547, Jul. 2018.

[18] E. Torelli, M. Manzano, S. K. Srivastava, and R. S. Marks, "DNA origami nanorobot fiber optic genosensor to TMV," Biosens. Bioelectron., vol. 99, no. February 2017, pp. 209-215, Jan. 2018.

[19] H. Pfeiffer et al., "Fiber optic SPR biosensing of DNA hybridization and DNA-protein interactions," Biosens. Bioelectron., vol. 25, no. 4, pp. 864-869, 2009.

[20] D. Daems, K. Knez, F. Delport, D. Spasic, and J. Lammertyn, "Real-time PCR melting analysis with fiber optic SPR enables multiplex DNA identification of bacteria,” Analyst, vol. 141, no. 6, pp. 1906-1911, 2016.

[21] W. Gong et al., "Experimental and theoretical investigation for surface plasmon resonance biosensor based on graphene/Au film/DPOF,” Opt. Express, vol. 27, no. 3, p. 3483, 2019.

[22] S. Jia, C. Bian, J. H. Tong, J. Z. Sun, and S. H. Xia, “A localized surface plasmon resonance DNA biosensor based on gold nanospheres coated on the tip of the fiber," Optoelectron. Lett., vol. 12, no. 2, pp. 157-160, 2016.

[23] S. Kaye et al., "Label-free detection of DNA hybridization with a compact LSPR-based fiber-optic sensor,” Analyst, vol. 142, no. 11, pp. 1974-1981, 2017.

[24] H. S. Jang, K. N. Park, J. P. Kim, and S. J. Sim, "Sensitive DNA biosensor based on a long-period grating formed on the sidepolished fiber surface,” Opt. Express, vol. 17, no. 5, p. 3855, 2009. H. M. R. Gonçalves et al., "Biosensor for label-free DNA quantification based on functionalized LPGs," Biosens. Bioelectron., vol. 84, pp. 30-36, 2016.

[26] M. Delgado-Pinar et al., "Oligonucleotide-Hybridization FiberOptic Biosensor Using a Narrow Bandwidth Long Period Grating," IEEE Sens. J., vol. 17, no. 17, pp. 5503-5509, 2017.

[27] D. Sun, T. Guo, and B. O. Guan, "Label-free detection of DNA hybridization using a reflective microfiber bragg grating biosensor with self-assembly technique,” J. Light. Technol., vol. 35, no. 16, pp. 3354-3359, 2017.

[28] D. Sun, T. Guo, Y. Ran, Y. Huang, and B. O. Guan, "In-situ DNA hybridization detection with a reflective microfiber grating biosensor,” Biosens. Bioelectron., vol. 61, pp. 541-546, 2014.

[29] K. L. Cooper, A. B. Bandara, Y. Wang, A. Wang, and T. J. Inzana, "Photonic biosensor assays to detect and distinguish subspecies of Francisella tularensis,” Sensors, vol. 11, no. 3, pp. 3004-3019, 2011.

[30] Y. Huang et al., "High-sensitivity DNA biosensor based on optical fiber taper interferometer coated with conjugated polymer tentacle,” Opt. Express, vol. 23, no. 21, p. 26962, 2015.

[31] Y. Huang, B. Yu, T. Guo, and B. O. Guan, "Ultrasensitive and in situ DNA detection in various $\mathrm{pH}$ environments based on a microfiber with a graphene oxide linking layer," RSC Adv., vol. 7, no. 22, pp. 13177-13183, 2017.

[32] S. Gao et al., "High-sensitivity DNA biosensor based on microfiber Sagnac interferometer,” Opt. Express, vol. 25, no. 12, p. 13305, 2017.

[33] N. H. Zainuddin, H. Y. Chee, M. Z. Ahmad, M. A. Mahdi, M. H. Abu Bakar, and M. H. Yaacob, "Sensitive Leptospira DNA detection using tapered optical fiber sensor," J. Biophotonics, vol. 11, no. 8, pp. 1-12, 2018.

[34] Y. Li, F. Fang, L. Yang, S. Tan, Z. Yan, and Q. Sun, "In-situ DNA hybridization detection based on a reflective microfiber probe," Opt. Express, vol. 28, no. 2, p. 970, 2020.

[35] M. I. Zibaii, Z. Taghipour, Z. Saeedian, H. Latifi, M. Gholami, and S. M. Hosseini, "Kinetic study for the hybridization of 25-mer DNA by nonadiabatic tapered optical fiber sensor," Opt. InfoBase Conf. Pap., vol. 8311, pp. 1-6, 2011.

[36] E. Kretschmann and H. Raether, "Radiative Decay of Non Radiative Surface Plasmons Excited by Light," Zeitschrift fur Naturforsch. Sect. A J. Phys. Sci., vol. 23, no. 12, pp. 2135-2136, 1968.

[37] R. C. Jorgenson and S. S. Yee, "A fiber-optic chemical sensor based on surface plasmon resonance," Sensors Actuators B. Chem., vol. 12, no. 3, pp. 213-220, 1993.

[38] A. Urrutia, I. Del Villar, P. Zubiate, and C. R. Zamarreño, “A Comprehensive Review of Optical Fiber Refractometers: Toward a
Standard Comparative Criterion," Laser Photonics Rev., vol. 13, no. 11, pp. 1-32, 2019.

[39] M. Xue, Q. Jiang, C. Zhang, and J. Lin, "A kind of biomolecular probe sensor based on TFBG surface plasma resonance," Photonic Sensors, vol. 5, no. 2, pp. 102-108, 2015.

[40] J. Wu, X. Zhang, B. Liu, H. Zhang, and B. Song, "Squaremicrofiber-integrated biosensor for label-free DNA hybridization detection," Sensors Actuators, B Chem., vol. 252, pp. 1125-1131, 2017.

[41] X. Zhang et al., "Label-free detection of DNA hybridization utilizing dual S-tapered thin-core fiber interferometer," J. Light. Technol., vol. 8724, no. c, 2018.

[42] F. Feng, W. Chen, D. Chen, W. Lin, and S. C. Chen, "In-situ ultrasensitive label-free DNA hybridization detection using optical fiber specklegram,” Sensors Actuators, B Chem., vol. 272, no. April, pp. 160-165, 2018.

[43] S. Niu, Q. Li, R. Ren, and S. Zhang, "Enzyme-enhanced fluorescence detection of DNA on etched optical fibers," Biosens. Bioelectron., vol. 24, no. 9, pp. 2943-2946, 2009.

[44] R. Xiao, Z. Rong, F. Long, and Q. Liu, "Portable evanescent wave fiber biosensor for highly sensitive detection of Shigella," Spectrochim. Acta - Part A Mol. Biomol. Spectrosc., vol. 132, pp. 15, 2014.

[45] J. Koppert et al., "Self-heating tilted fiber Bragg grating device for melt curve analysis of solid-phase DNA hybridization and thermal cycling," Anal. Bioanal. Chem., vol. 411, no. 26, pp. 6813-6823, 2019.

[46] E. Torelli, M. Manzano, and R. S. Marks, "Chemiluminescent optical fibre genosensor for porcine meat detection," Sensors Actuators, B Chem., vol. 247, pp. 868-874, 2017.

[47] R. D’Agata, M. C. Giuffrida, and G. Spoto, "Peptide Nucleic AcidBased Biosensors for Cancer Diagnosis,” Molecules, vol. 22, no. 11, pp. 1-15, 2017.

[48] E. Coscelli et al., "Toward a highly specific DNA biosensor: PNAmodified suspended-core photonic crystal fibers," IEEE J. Sel. Top. Quantum Electron., vol. 16, no. 4, pp. 967-972, 2010.

[49] M. Sozzi et al., "Long period grating-based fiber optic sensor for label-free DNA detection,” 2011 Int. Work. Biophotonics, Biophotonics 2011, pp. 11-13, 2011.

[50] A. Bertucci et al., "Label-free DNA biosensor based on a peptide nucleic acid-functionalized microstructured optical fiber-Bragg grating,” J. Biomed. Opt., vol. 18, no. 5, p. 057004, 2013.

[51] A. Candiani et al., "Optical fiber ring cavity sensor for label-free DNA detection,” IEEE J. Sel. Top. Quantum Electron., vol. 18, no. 3, pp. 1176-1183, 2012.

[52] A. Bertucci et al., "Detection of unamplified genomic DNA by a PNA-based microstructured optical fiber (MOF) Bragg-grating optofluidic system,” Biosens. Bioelectron., vol. 63, pp. 248-254, 2015.

[53] S. Tyagi and F. R. Kramer, "Molecular Beacons: Probes that Fluoresce upon Hybridization," Nat. Biotechnol., vol. 14, no. 3, pp. 303-308, Mar. 1996.

[54] X. Liu and W. Tan, "A Fiber-Optic Evanescent Wave DNA Biosensor Based on Novel Molecular Beacons," Anal. Chem., vol. 71, no. 22, pp. 5054-5059, Nov. 1999.

[55] A. Giannetti et al., "Optical Fiber Nanotips Coated with Molecular Beacons for DNA Detection,” Sensors, vol. 15, no. 5, pp. 96669680, Apr. 2015.

[56] L. V. Nguyen, S. C. Warren-Smith, A. Cooper, and T. M. Monro, "Molecular beacons immobilized within suspended core optical fiber for specific DNA detection," Opt. Express, vol. 20, no. 28, p. 29378, 2012.

[57] Y. Yonamine et al., "Supramolecular 1-D polymerization of DNA origami through a dynamic process at the 2-dimensionally confined air-water interface," Phys. Chem. Chem. Phys., vol. 18, no. 18, pp. 12576-12581, 2016.

[58] Y. R. Yang, Y. Liu, and H. Yan, "DNA Nanostructures as Programmable Biomolecular Scaffolds,” Bioconjug. Chem., vol. 26, no. 8, pp. 1381-1395, Aug. 2015.

[59] A. Kuzuya and Y. Ohya, "Nanomechanical Molecular Devices made of DNA Origami," Acc. Chem. Res., vol. 47, no. 6, pp. 17421749, Jun. 2014.

[60] C. Angell, S. Xie, L. Zhang, and Y. Chen, "DNA Nanotechnology for Precise Control over Drug Delivery and Gene Therapy,” Small, vol. 12, no. 9, pp. 1117-1132, Mar. 2016. 
[61] A. R. Chandrasekaran, H. Wady, and H. K. K. Subramanian, "Nucleic Acid Nanostructures for Chemical and Biological Sensing,” Small, vol. 12, no. 20, pp. 2689-2700, 2016.

[62] E. Torelli, M. Manzano, S. K. Srivastava, and R. S. Marks, "DNA origami nanorobot fiber optic genosensor to TMV," Biosens. Bioelectron., vol. 99, no. February 2017, pp. 209-215, Jan. 2018.

[63] D. Daems, W. Pfeifer, I. Rutten, B. Saccà, D. Spasic, and J. Lammertyn, "Three-Dimensional DNA Origami as Programmable Anchoring Points for Bioreceptors in Fiber Optic Surface Plasmon Resonance Biosensing,” ACS Appl. Mater. Interfaces, vol. 10, no. 28, pp. 23539-23547, Jul. 2018.

[64] X. Zhu, R. Wang, K. Xia, X. Zhou, and H. Shi, "Nucleic acid functionalized fiber optic probes for sensing in evanescent wave: optimization and application," RSC Adv., vol. 9, no. 4, pp. 23162324, 2019.

[65] W. Hu, Y. Huang, C. Chen, Y. Liu, T. Guo, and B. O. Guan, "Highly sensitive detection of dopamine using a graphene functionalized plasmonic fiber-optic sensor with aptamer conformational amplification,” Sensors Actuators, B Chem., vol. 264, pp. 440-447, 2018.

[66] M. I. Zibaii et al., "Nonadiabatic tapered optical fiber sensor for measuring interaction nicotine with DNA," 21st Int. Conf. Opt. Fiber Sensors, vol. 7753, p. 77536C, 2011.

[67] N. Cennamo et al., "An easy way to realize SPR aptasensor: A multimode plastic optical fiber platform for cancer biomarkers detection,” Talanta, vol. 140, pp. 88-95, 2015.

[68] J. Albert, S. Lepinay, C. Caucheteur, and M. C. DeRosa, "High resolution grating-assisted surface plasmon resonance fiber optic aptasensor,” Methods, vol. 63, no. 3, pp. 239-254, 2013.

[69] K. Ye et al., "Development of a chemiluminescent DNA fibre optic genosensor to Hepatitis A Virus (HAV),” Talanta, vol. 174, no. June, pp. 401-408, 2017.

[70] S. Qian et al., "Boronic Acid Functionalized Au Nanoparticles for Selective MicroRNA Signal Amplification in Fiber-Optic Surface Plasmon Resonance Sensing System,” ACS Sensors, vol. 3, no. 5, pp. 929-935, 2018.

[71] S. Jia, C. Bian, J. Sun, J. Tong, and S. Xia, “A wavelengthmodulated localized surface plasmon resonance (LSPR) optical fiber sensor for sensitive detection of mercury(II) ion by gold nanoparticles-DNA conjugates,” Biosens. Bioelectron., vol. 114, no. April, pp. 15-21, 2018.

[72] N. Polley, P. K. Sarkar, S. Chakrabarti, P. Lemmens, and S. K. Pal, "DNA Biomaterial Based Fiber Optic Sensor: Characterization and Application for Monitoring in situ Mercury Pollution," ChemistrySelect, vol. 1, no. 11, pp. 2916-2922, Jul. 2016.

[73] S. Hong et al., "Thermo-optic characteristic of DNA thin solid film and its application as a biocompatible optical fiber temperature sensor," Opt. Lett., vol. 42, no. 10, p. 1943, May 2017.

[74] P. K. Sarkar et al., "DNA-based fiber optic sensor for direct in-vivo measurement of oxidative stress," Sensors Actuators, B Chem., vol. 255, pp. 2194-2202, 2018.

[75] F. Cecchini, M. Manzano, Y. Mandabi, E. Perelman, and R. S. Marks, "Chemiluminescent DNA optical fibre sensor for Brettanomyces bruxellensis detection,” J. Biotechnol., vol. 157, no. 1, pp. 25-30, 2012.

[76] D. Song, R. Yang, S. Fang, Y. Liu, and F. Long, “A FRET-based dual-color evanescent wave optical fiber aptasensor for simultaneous fluorometric determination of aflatoxin M1 and ochratoxin A," Microchim. Acta, vol. 185, no. 11, pp. 1-10, 2018.

[77] N. Yildirim, F. Long, M. He, H.-C. Shi, and A. Z. Gu, "A portable optic fiber aptasensor for sensitive, specific and rapid detection of bisphenol-A in water samples," Environ. Sci. Process. Impacts, vol. 16, no. 6, pp. 1379-1386, 2014.

[78] S. Hong et al., "Thermo-optic characteristic of DNA thin solid film and its application as a biocompatible optical fiber temperature sensor," Opt. Lett., vol. 42, no. 10, p. 1943, May 2017.

[79] P. Zubiate, C. R. Zamarreño, P. Sánchez, I. R. Matias, and F. J. Arregui, "High sensitive and selective C-reactive protein detection by means of lossy mode resonance based optical fiber devices," Biosens. Bioelectron., vol. 93, pp. 176-181, Jul. 2017.

[80] L. Razquin, C. R. Zamarreno, F. J. Munoz, I. R. Matias, and F. J. Arregui, "Thrombin detection by means of an aptamer based sensitive coating fabricated onto LMR-based optical fiber refractometer," Proc. IEEE Sensors, pp. 3-6, 2012.

[81] Y. Li, R. Tian, X. Zheng, and R. Huang, “Amplified electrochemical detection of nucleic acid hybridization via selective preconcentration of unmodified gold nanoparticles," Anal. Chim. Acta, vol. 934, pp. 59-65, 2016.

[82] J. Albert, S. Lepinay, C. Caucheteur, and M. C. DeRosa, "High resolution grating-assisted surface plasmon resonance fiber optic aptasensor,” Methods, vol. 63, no. 3, pp. 239-254, 2013.

[83] W. Hu, Y. Huang, C. Chen, Y. Liu, T. Guo, and B. O. Guan, "Highly sensitive detection of dopamine using a graphene functionalized plasmonic fiber-optic sensor with aptamer conformational amplification," Sensors Actuators, B Chem., vol. 264, pp. 440-447, 2018.

[84] N. Cennamo et al., "An easy way to realize SPR aptasensor: A multimode plastic optical fiber platform for cancer biomarkers detection,” Talanta, vol. 140, pp. 88-95, 2015.

[85] S. Jia, C. Bian, J. Sun, J. Tong, and S. Xia, "A wavelengthmodulated localized surface plasmon resonance (LSPR) optical fiber sensor for sensitive detection of mercury(II) ion by gold nanoparticles-DNA conjugates,” Biosens. Bioelectron., vol. 114, no. April, pp. 15-21, 2018.

[86] K. Ye et al., "Development of a chemiluminescent DNA fibre optic genosensor to Hepatitis A Virus (HAV)," Talanta, vol. 174, no. June, pp. 401-408, 2017.

[87] N. Polley, P. K. Sarkar, S. Chakrabarti, P. Lemmens, and S. K. Pal, "DNA Biomaterial Based Fiber Optic Sensor: Characterization and Application for Monitoring in situ Mercury Pollution," ChemistrySelect, vol. 1, no. 11, pp. 2916-2922, Jul. 2016.

[88] E. Torelli, M. Manzano, and R. S. Marks, "Chemiluminescent optical fibre genosensor for porcine meat detection," Sensors Actuators, B Chem., vol. 247, pp. 868-874, 2017.

[89] P. K. Sarkar et al., "DNA-based fiber optic sensor for direct in-vivo measurement of oxidative stress," Sensors Actuators, B Chem., vol. 255, pp. 2194-2202, 2018.

[90] S. M. Tripathi et al., "Gold coated dual-resonance long-period fiber gratings (DR-LPFG) based aptasensor for cyanobacterial toxin detection," Sensing and Bio-Sensing Research, vol. 25, pp. 1-8, 2019. 